\title{
Quantum and Thermal Fluctuations in the Ising Chains: A Case Study of Spin-Two System
}

\author{
S. Ehika*, O. J. Ataman, S.E. Iyayi \\ Department of Physics, Ambrose Alli University, Ekpoma, Edo State, NIGERIA.
}

\begin{abstract}
For a system at absolute zero temperature, all thermal fluctuations are frozen out, while quantum fluctuations prevail. These microscopic quantum fluctuations can induce a quantum phase transition $(Q P T)$ in the ground state of a many-body system when the relative strength of two competing energy terms is varied across a critical value. This paper presents a detailed and quantitative study of the magnetic properties of Ising two-spin system at zero temperature in the presence of longitudinal, transverse and mixed fields, and also at finite temperatures. At the critical longitudinal magnetic field, the system is found to undergo a QPT to a ferromagnetic state. For this system, an infinitesimal departure from zero transverse field is found to trigger off QPT and also remove the degeneracies that persisted even in the presence of longitudinal field. These quantum fluctuations, leading to QPT are more evident in the mixed field than the separate effect of longitudinal and transverse fields. The observed quantum effects at zero temperature for this system are found to be completely wiped out at finite temperatures due to thermal fluctuations. A temperature increase favours antiferromagnetic alignment of spins, while a decrease in temperature favours ferromagnetic alignment.
\end{abstract}

Keywords: Quantum phase transition, thermal fluctuations, quantum fluctuations, Ising systems, longitudinal field, and transverse field.

\section{Introduction}

We all observe phase transitions in our daily lives, with hardly a second thought. When we boil water for a cup of tea, we observe that the water remain quiescent until it reaches a certain temperature $\left(100^{\circ} \mathrm{C}\right)$, and then bubbles appear vigorously until all the water has turned to steam. We have watched the melting away of snow gathered overnight when temperatures rise during the day. The more adventurous among us may have heated an iron magnet to a temperature of about $760^{\circ} \mathrm{C}$ and noted the disappearance of its magnetism. Familiar and ubiquitous as these and many related phenomena are, a little reflection shows that they are quite mysterious and not easy to understand. Indeed, the reasons for these observed phenomena did not emerge until the middle of the $20^{\text {th }}$ century. Although, much has been understood since then, active research continues. Ice and water both consist of molecules of $\mathrm{H}_{2} \mathrm{O}$, and we can look up all the physical parameters of a single molecule, and of the interaction between a pair of molecules, in standard reference texts. However, no detailed study of this information prepares us for the dramatic change that occurs at $0^{\circ} \mathrm{C}$. Below $0^{\circ} \mathrm{C}$, the $\mathrm{H}_{2} \mathrm{O}$ molecules of ice are arranged in a regular crystalline lattice, and each $\mathrm{H}_{2} \mathrm{O}$ molecule hardly strays from its own lattice site. Above $0^{\circ} \mathrm{C}$, we obtain liquid water, in which all the molecules are moving freely throughout the liquid container at high speeds. Why do $10^{23} \mathrm{H}_{2} \mathrm{O}$ molecules co-operatively "decide" to become mobile at a certain temperature, leading to the phase transition from ice to water?

In Physics, a quantum phase transition (QPT) is a phase transition between different quantum phases of matter at absolute zero temperature [1-8]. Contrary to classical phase transitions, quantum phase transitions can only be accessed by varying a physical parameter such as magnetic field or pressure at absolute zero temperature. The transition describes an abrupt change in the ground state of a many-body system due to its quantum fluctuations. At the critical point where the quantum phase transition (QPT) occurs, the ground state of the system undergoes a qualitative change in some of its properties [5,6]. QPT can be described as second order phase transition. On the other hand, classical phase transitions are driven by a competition between the energy of a system and the entropy of its thermal fluctuations. A classical system does not have entropy at absolute zero temperature and therefore no phase transition can occur. Their order is determined by the first discontinuous derivative of a thermodynamic potential. A phase transition from water to ice, for example, involves latent heat (a discontinuity of the heat capacity) and is of first order.

Among the various systems, one dimensional (1D) and quasi-one dimensional systems are fantastic playground for quantum phase transitions (QPTs), with rather unique properties $[3,5,6,8,10,11,12]$. There are various reasons for this special behaviour. Firstly, contrary to their higher dimensional counterparts, interactions play a major role in 1D particles. These interactions transform any individual motion of the particles into a collective one. Secondly, in 1D particles quantum and thermal fluctuations are pushed to a maximum, and prevent the breaking of continuous symmetries, making simple mean-field Physics inapplicable. The combination of these two effects leads to a very special universality class for interacting quantum systems, known as Luttinger liquids (LLs) [12-14]. The important point is that LL is taken to be in a critical phase in which correlations decrease at zero temperature, as power laws of space and time. This makes the system extremely fragile to external perturbations and leads to a host of QPTs. Examples of such perturbations are the effects of large Coulomb repulsion which leads to a Mott transition [15], and disorder that leads to localized phases such as Anderson localization or the Bose glass [16-17]. Each of these transitions is characterized by a quantum critical point (QCP) that can be computed from LL theory [18-20].

The study of the field-induced effects in low-dimensional quantum spin systems has been attracting much interest from theoretical and experimental points of view $[1,3,5,6,8,11]$. For example, the behaviour of the one-dimensional antiferromagnetic XXZ model in a transverse magnetic field is drastically different in comparison with the case of the longitudinal field [8,21, and 22]. The one-dimensional (1D) Ising chain in transverse field is perhaps the most-studied 
theoretical paradigm for a quantum phase transition [10, 22]. More so, in a class of one-dimensional magnetic systems, QPT has also been shown to be associated with a change of entanglement [23-24]. This entanglement is known to exhibit scaling behaviour in the vicinity of the transition point [25]. This behaviour was discussed in detail for the Heisenberg model by Osborne and Nielsen in ref. [25].

Quantum phase transitions are relevant in quantum computing, e.g., adiabatic quantum computing [26-27] and quantum estimation [28-29]. Interesting phenomena related to QPTs have recently been experimentally observed in various systems, such as Cuprate Superconductors [30-31], heavy fermions and Bose-Einstein condensates [32], magnetic insulators [33-34] and Jaynes-Cummings-Hubbard (JCH) system [35]. The aim of this work is to study the effect of external magnetic fields, namely longitudinal, transverse and mixed fields at zero and finite temperatures on the ground state properties of the Ising spin chains, using two-spin system as a case study. The results obtain for this simple system will be useful for the advance study of complicated systems. This study is also expected to provide further insight into the behaviour of one and quasi-one dimensional systems subject to varying magnetic fields at zero and finite temperatures.

The remainder of this paper is organized as follows. In section II, we give a brief description of the Ising model. Section III investigates the effect of zero field and zero temperature on Ising spin-two system. Section IV investigates the effect of longitudinal field on the system. Section V investigates the effect of transverse field on the system. Section VI investigates the effect of mixed field on the system. Section VII investigates the response of this system to finite temperatures. We present and discuss results in section VIII. We conclude in section IX.

\section{Ising model}

The Ising model is the simplest theoretical description of ferromagnetism. This model was invented by Wilhelm Lenz in 1920 and named after Ernst Ising, a student of Lenz who chose the model as the subject of his doctoral dissertation. The model consists of discrete variables called spins that are arranged on a lattice. The antiferromagnetic version of this model in one dimension reads:

$H=J \sum_{i=1}^{N} S_{i}^{z} S_{i+1}^{z}-h_{z} \sum_{i} S_{i}^{z}-h_{x} \sum_{i} S_{i}^{x}$,

where $J$ is the superexchange coupling parameter between nearest neighbour spins. The symbols $S_{i}^{z}$ and $S_{i}^{x}$ are the diagonal and off diagonal spin operators respectively. These operators act in the reduced Hilbert space of no doubly occupied sites. The longitudinal and transverse field are respectively denoted by $h_{z}$ and $h_{x}$. The Ising model can be recovered from the Heisenberg model by switching off the spin fluctuation term, i.e. $S_{i}^{+} S_{i+1}^{-}+S_{i}^{-} S_{i+1}^{+}=0$. In terms of creation and annihilation operator, the form in (1) can be written as:

$$
\begin{aligned}
& H=\frac{J}{4} \sum_{i}\left(c_{i \uparrow}^{\dagger} c_{i \uparrow} c_{(i+1) \uparrow}^{\dagger} c_{(i+1) \uparrow}-c_{i \uparrow}^{\dagger} c_{i \uparrow} c_{(i+1) \downarrow}^{\dagger} c_{(i+1) \downarrow}-c_{i \downarrow}^{\dagger} c_{i \downarrow} c_{(i+1) \uparrow}^{\dagger} c_{(i+1) \uparrow}+c_{i \downarrow}^{\dagger} c_{i \downarrow} c_{(i+1) \downarrow}^{\dagger} c_{(i+1) \downarrow}\right) \\
& -1 / 2 h_{z} \sum_{i}\left(c_{i \uparrow}^{\dagger} c_{i \uparrow}-c_{i \downarrow}^{\dagger} c_{i \downarrow}+c_{(i+1) \uparrow}^{\dagger} c_{(i+1) \uparrow}-c_{(i+1) \downarrow}^{\dagger} c_{(i+1) \downarrow}\right) \\
& +h_{x} \sum_{i}\left(c_{i \uparrow}^{\dagger} c_{i \downarrow}+c_{i \downarrow}^{\dagger} c_{1 \uparrow}+c_{(i+1) \uparrow}^{\dagger} c_{(i+1) \downarrow}+c_{(i+1) \downarrow}^{\dagger} c_{(i+1) \uparrow}\right)
\end{aligned}
$$

Here, summation over nearest neighbour is explicit

\section{Spin-two Ising system in zero field and zero temperature}

This section will examine the nature of the ground state of the simplest possible Ising cluster (i.e. the spin-1/2 two site dimer) in zero field. Periodic boundary conditions (PBC) is imposed on the spins so that $S_{N+1}^{z}=S_{1}^{z}$. Thus, the topology of the spin space is that of a circle as shown in Fig.1.

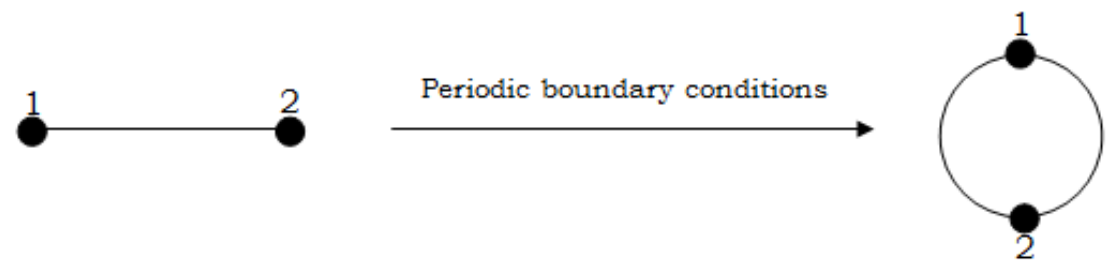

Fig.1. A two -site chain. The topology is that of a circle on the application of periodic boundary conditions.

The Hamiltonian of this system in the absence of external magnetic field is given by

$$
H_{0}=\frac{J}{4}\left(c_{1 \uparrow}^{\dagger} c_{1 \uparrow} c_{2 \uparrow}^{\dagger} c_{2 \uparrow}-c_{1 \uparrow}^{\dagger} c_{1 \uparrow} c_{2 \downarrow}^{\dagger} c_{2 \downarrow}-c_{1 \downarrow}^{\dagger} c_{1 \downarrow} c_{2 \uparrow}^{\dagger} c_{2 \uparrow}+c_{1 \downarrow}^{\dagger} c_{1 \downarrow} c_{2 \downarrow}^{\dagger} c_{2 \downarrow}\right)
$$


Since a spin have two configurations in space (i.e. spin up or spin down) and the exclusion of doubly occupied site is understood, the size of the Hilbert space of an Ising system with $\mathrm{N}$ spins is $2^{\mathrm{N}}$. Hence, for two-spin system, the size of the Hilbert space is 4 . The basis states are:

$$
|1\rangle=|1 \uparrow, 2 \uparrow\rangle,|2\rangle=|1 \downarrow, 2 \downarrow\rangle,|3\rangle=|1 \uparrow, 2 \downarrow\rangle,|4\rangle=|1 \downarrow 2 \uparrow\rangle
$$

It is possible to construct a matrix representation of the Hamiltonian by using $H_{0}$ to act on each of these basis states. Thus,

$$
\begin{aligned}
& H_{0}|1\rangle=H_{0}|1 \uparrow, 2 \uparrow\rangle=\frac{J}{4}|1 \uparrow, 2 \uparrow\rangle \\
& H_{0}|2\rangle=H_{0}|1 \downarrow, 2 \downarrow\rangle=\frac{J}{4}|1 \downarrow, 2 \downarrow\rangle \\
& H_{0}|3\rangle=H_{0}|1 \uparrow, 2 \downarrow\rangle=-\frac{J}{4}|1 \uparrow, 2 \downarrow\rangle \\
& H_{0}|4\rangle=H_{0}|1 \downarrow 2 \uparrow\rangle=-\frac{J}{4}|1 \downarrow 2 \uparrow\rangle
\end{aligned}
$$

These operations will lead to the matrix representation

$$
H_{0}=\frac{J}{4}\left[\begin{array}{cccc}
1 & 0 & 0 & 0 \\
0 & 1 & 0 & 0 \\
0 & 0 & -1 & 0 \\
0 & 0 & 0 & -1
\end{array}\right]
$$

At a glance, the Hamiltonian is observed to be block-diagonal with respect to subspaces $S_{\text {tot }}^{z}=1,-1$ and 0 , with two-fold degeneracy in both the ground and excited states.

\section{Effect Of External Longitudinal Magnetic Field}

In the presence of the longitudinal field, the Ising Hamiltonian for two-spin system, expressed in the second quantized form is given by:

$$
H_{h_{z}}=H_{0}+H_{z}
$$

where $H_{z}$ is given by:

$$
H_{z}=-\vec{h}_{z}\left(S_{1}^{z}+S_{2}^{z}\right)=-h_{z} / 2\left(c_{1 \uparrow}^{\dagger} c_{1 \uparrow}-c_{1 \downarrow}^{\dagger} c_{1 \downarrow}+c_{2 \uparrow}^{\dagger} c_{2 \uparrow}-c_{2 \downarrow}^{\dagger} c_{2 \downarrow}\right)
$$

Therefore, the Hamiltonian matrix in the presence of external longitudinal magnetic field gives:

$$
H_{h_{z}}=\left[\begin{array}{cccc}
J / 4-h_{z} & 0 & 0 & 0 \\
0 & h_{z}+J / 4 & 0 & 0 \\
0 & 0 & -J / 4 & 0 \\
0 & 0 & 0 & -J / 4
\end{array}\right]
$$

The eigen values of this matrix are: $\lambda_{1}=-J / 4, \lambda_{2}=-J / 4, \lambda_{3}=(J / 4)-h_{z}$ and $\lambda_{4}=(J / 4)+h_{z}$

Observe that the external longitudinal field does not have effect on antiferromagnetic states. At a glance, we see that the two-fold degenerate triplet state at zero magnetic field splits into two distinct states as the magnetic field is applied. The energy levels per magnetic coupling constant arising from (7) can be constructed as follows:

$$
\left.E_{1}=E_{2}=\lambda_{1} / J=-0.25, E_{3}=\lambda_{3} / J=0.25-\left(h_{z} / J\right), E_{4}=\lambda_{4} / J=0.25+\left(h_{z} / J\right)\right\}
$$

The competing energies for this system are the two-fold degenerate energy levels (i.e. $E_{1}$ and $E_{2}$ ) and the excited energy level $E_{3}$. The energy dependence of this system with longitudinal field is shown in Table 1 . This dependence and energy level crossing is illustrated in Fig. 2, while Fig. 3 captures the quantum of magnetization.

\section{Effect of external transverse magnetic field}

The Hamiltonian for two-spin Ising system in the presence of transverse field gives:

$$
H_{h_{x}}=H_{0}+H_{x}
$$

Here, the Hamiltonian $H_{x}$ is given by: 
$H_{x}=-\vec{h}_{x}\left(S_{1}^{x}+S_{2}^{x}\right)=-h_{x} / 2\left(c_{1 \uparrow}^{\dagger} c_{1 \downarrow}+c_{1 \downarrow}^{\dagger} c_{1 \uparrow}+c_{2 \uparrow}^{\dagger} c_{2 \downarrow}+c_{2 \downarrow}^{\dagger} c_{2 \uparrow}\right)$

The Hamiltonian matrix in the presence of an external transverse magnetic field gives:

$$
H_{h_{x}}=\left[\begin{array}{cccc}
J / 4 & 0 & -h_{x} / 2 & -h_{x} / 2 \\
0 & J / 4 & -h_{x} / 2 & -h_{x} / 2 \\
-h_{x} / 2 & -h_{x} / 2 & -J / 4 & 0 \\
-h_{x} / 2 & -h_{x} / 2 & 0 & -J / 4
\end{array}\right]
$$

The complete diagonalization of the Hamiltonian matrix in (11) gives the following eigenvalues:

$$
\left.\lambda_{1}=-\frac{J}{4}, \lambda_{2}=\frac{J}{4}, \lambda_{3}=-\frac{1}{4} \sqrt{16 h_{x}^{2}+J^{2}}, \lambda_{4}=\frac{1}{4} \sqrt{16 h_{x}^{2}+J^{2}}\right\}
$$

These eigenvalues in (12) can be recast as follows:

$$
\left.E_{1}=\frac{\lambda_{1}}{J}=-\frac{1}{4}, E_{2}=\frac{\lambda_{2}}{J}=\frac{1}{4}, E_{3}=\frac{\lambda_{3}}{J}=-\frac{1}{4} \sqrt{16\left(\frac{h_{x}}{J}\right)^{2}+1}, E_{4}=\frac{\lambda_{4}}{J}=\frac{1}{4} \sqrt{16\left(\frac{h_{x}}{J}\right)^{2}+1}\right\}
$$

It is obvious that the twofold degenerate ground state that persists even in the presence of longitudinal field is completely removed by the transverse field. The two competing energies are now $E_{1}$ and $E_{3}$. The dynamics of the spins in the corresponding eigen states at zero temperature is completely driven by quantum fluctuations. The variations of the ground and excited state energies with the transverse field are presented in Table 2 and Fig.4.

\section{Effect of mixed field}

The Hamiltonian for mixed field (i.e. involving both longitudinal and transverse fields) is given by

$$
H=H_{h}+H_{0}
$$

where $H_{h}$ is given by

$H_{h}=H_{z}+H_{x}$

For two-spin system, (15) gives

$$
H_{h}=-h_{z} / 2\left(c_{1 \uparrow}^{\dagger} c_{1 \uparrow}-c_{1 \downarrow}^{\dagger} c_{1 \downarrow}+c_{2 \uparrow}^{\dagger} c_{2 \uparrow}-c_{2 \downarrow}^{\dagger} c_{2 \downarrow}\right)-h_{x} / 2\left(c_{1 \uparrow}^{\dagger} c_{1 \downarrow}+c_{1 \downarrow}^{\dagger} c_{1 \uparrow}+c_{2 \uparrow}^{\dagger} c_{2 \downarrow}+c_{2 \downarrow}^{\dagger} c_{2 \uparrow}\right)
$$

The interaction of this mixed field with the two-spin system gives the following

$$
\begin{aligned}
& H_{h}|1 \uparrow, 2 \uparrow\rangle=-\frac{h_{x}}{2}[|1 \uparrow, 2 \downarrow\rangle+|1 \downarrow, 2 \uparrow\rangle]-\frac{h_{z}}{2}|1 \uparrow, 2 \uparrow\rangle=-\frac{h_{x}}{2}[|3\rangle+|4\rangle]-h_{z}|1\rangle \\
& H_{h}|1 \downarrow, 2 \downarrow\rangle=-\frac{h_{x}}{2}[|1 \downarrow, 2 \uparrow\rangle+|1 \uparrow, 2 \downarrow\rangle]+h_{z}|1 \downarrow, 2 \downarrow\rangle=-\frac{h_{x}}{2}[|4\rangle+|3\rangle]+h_{z}|2\rangle \\
& H_{h}|1 \uparrow, 2 \downarrow\rangle=-\frac{h_{x}}{2}[|1 \uparrow, 2 \uparrow\rangle+|1 \downarrow, 2 \downarrow\rangle]-\frac{h_{z}}{2}[|1 \uparrow, 2 \downarrow\rangle-|1 \uparrow, 2 \downarrow\rangle]=-\frac{h_{x}}{2}[|1\rangle+|2\rangle] \\
& H_{h}|1 \downarrow, 2 \uparrow\rangle=-\frac{h_{x}}{2}[|1 \downarrow, 2 \downarrow\rangle+|1 \uparrow, 2 \uparrow\rangle]-\frac{h_{z}}{2}[|1 \downarrow, 2 \uparrow\rangle-|1 \downarrow, 2 \uparrow\rangle]=-\frac{h_{x}}{2}[|2\rangle+|1\rangle]
\end{aligned}
$$

The Hamiltonian matrix arising from the interaction of this mixed field with two-spin system gives:

$$
H=\left[\begin{array}{ccrc}
\frac{J}{4}-h_{z} & 0 & -\frac{h_{x}}{2} & -\frac{h_{x}}{2} \\
0 & \frac{J}{4}+h_{z} & -\frac{h_{x}}{2} & -\frac{h_{x}}{2} \\
-\frac{h_{x}}{2} & -\frac{h_{x}}{2} & -\frac{J}{4} & 0 \\
-\frac{h_{x}}{2} & -\frac{h_{x}}{2} & 0 & -\frac{J}{4}
\end{array}\right]
$$

The variations in the excited and ground state energies when $h_{x}$ and $h_{z}$ are simultaneously varied are shown in Table 3 . By keeping $\mathrm{h}_{\mathrm{z}}$ at its critical field $\left(\mathrm{h}_{\mathrm{z}}=0.5\right)$, the response of the spins to variations in $h_{x}$ is also investigated as presented in 
Table 4. The quantum spin fluctuations at the ground state when either $\mathrm{h}_{\mathrm{z}}$ or $h_{x}$ is fixed and the other varied are presented in Table 5 .

VII. Effect of finite temperatures on two-spin system

At finite temperatures, $T$ enters the Hamiltonian through the uniform magnetization given by

$$
m\left(h_{z}, T\right)=\frac{\sum_{i} S_{i}^{z} \exp \left(-\beta E_{i}\right)}{\sum_{i} \exp \left(-\beta E_{i}\right)}
$$

For the case of the two-spin Ising system, Eq. (18) is expanded to give

$$
m\left(h_{z}, T\right)=\frac{\exp \beta h_{z}-\exp \left(-\beta h_{z}\right)}{\exp \beta h_{z}+\exp \left(-\beta h_{z}\right)+2 \exp \beta(J / 2)}
$$

Finally, we have

$$
m(h, T)=\frac{2 \operatorname{Sinh}\left(\beta h_{z}\right)}{2 \operatorname{Cosh}\left(\beta h_{z}\right)+2 \exp \beta(J / 2)}
$$

Magnetic field dependence of the uniform magnetization calculated for fixed $\mathrm{T}=0.04 \mathrm{~J}, 0.08 \mathrm{~J}, 0.1 \mathrm{~J}$ and $0.4 \mathrm{~J}$ is shown in Table 6 and also illustrated Fig.5. Temperature dependence of the uniform magnetization calculated for fixed $\mathrm{h}_{\mathrm{z}}=0.8 \mathrm{~J}, 1.6 \mathrm{~J}, 2 \mathrm{~J}, 2.5 \mathrm{~J}$ is presented in Table 7 and also captured in Fig. 6.

\section{Results and discussion}

This section presents and discusses the results obtained for two-spin Ising system in the presence of external fields at zero temperature and finite temperatures. The variations of the energies of the two-spin system with finite longitudinal fields at zero temperature are shown in Table 1. As observed from Table 1, the two-fold degenerate ground state energy $E_{1} / J=E_{2} / J=-0.25$ unaffected by the field. This is because the configurations of the spins in this state are antiferromagnetic which do not respond to external longitudinal magnetic field. The energy $E_{4} / J$ exhibits a linear behaviour with $h_{z}$, while $E_{3} / J$ exhibits an inverse behaviour with $h_{z}$ as shown in Fig. 2. At a critical field of $h_{z c}=J / 2$ (or $h_{z c} / J=1 / 2$ ), there is a cross over where the $S_{t o t}^{z}=1$ state becomes the ground state, and therefore magnetization jumps from 0 to 1 as shown in Fig.3. In other words, the system undergoes a quantum phase transition (QPT) from antiferromagnetic ground state to ferromagnetic ground state. This energy level crossing changes the ground state from $E_{1} / J=E_{2} / J$ to $E_{3} / J$.

Next, we investigate the response of the spins to external transverse field at $h_{z}=0$ and $T=0$. It is observed that quantum fluctuation which gives rise to QPT is immediately triggered at infinitesimal transverse field. The degeneracy that persisted even at finite longitudinal fields is completely wiped out at very small transverse field as shown in Table 2 . An avoided level-crossing between the ground state with energy $E_{1}$ and the competing excited state with energy $E_{3}$ is observed as shown in Fig. 4. The ground state energy now shifts from $E_{1}$ to $E_{3}$. This implies a shift in ground state from antiferromagnet to ferromagnet.

For the case of the mixed field, we first study the response of the spin to simultaneous variations in longitudinal and transverse fields at $T=0$. As seen from Table 3, the simultaneous variations of $h_{x}$ and $h_{z}$ disrupt the antiferromagnetic alignment of spin more compare to the separate effect of $h_{x}$ and $h_{z}$ as captured in Table 1 and 2 respectively. A further study is carried out by keeping the longitudinal field at its critical value $\left(h_{z}=0.5\right)$ and then varying $h_{x}$. This study which is presented in Table 4 enables us to visualize the rapid lifting up of the degeneracy by $h_{x}$ that persisted even in the presence of $h_{z}$. The removal of this degeneracy by transverse field is reflected in the increase of the energy $\mathrm{E}_{3}$ and the subsequent decrease of the energy $\mathrm{E}_{1}$. This transfer of energy from $E_{1}$ to $E_{3}$ implies a shift of ground state energy from $E_{3}$ to $E_{1}$. The spins of the electrons in $E_{1}$ are expected to be ferromagnetically aligned. The comparison between the response of the Ising two-spin system to $h_{x}$ and its response to $h_{z}$ is drawn by keeping either $h_{z}$ or $h_{x}$ constant at 0.1 and varying the other. This study which is presented in Table 5 reveals that the two-spin system responds faster to $h_{x}$ than $h_{z}$. This observation further provides evidence for the fast disappearance of the antiferromagnetic ground state and the emergence of a ferromagnetic ground state in the presence of a transverse field. 
However, by comparing Tables 3 and 5, it can be seen that the simultaneous variations of $h_{x}$ and $h_{z}$ trigger off quantum phase transition more quickly than the independent variations of $h_{x}$ and $h_{z}$. At finite temperatures, the quantum jump observed in Fig. 3 is smoothened out. This is because they are wiped out by thermal fluctuations. The magnetic field and temperature dependence of the uniform magnetization as calculated from (18) is presented in Table 6 and 7 respectively. The temperature dependence of the uniform magnetization depends on the strength of the applied magnetic field. If the applied field is less than the critical field strength, the zero-temperature magnetization vanishes, and $m(h, T)$ is thermally activated, i.e. $m(h, T) \propto \exp (\beta h)[\exp (\beta h)+2 \exp (\beta J / 2)]^{-1}$. On the other hand, if the applied field exceeds the critical field strength at zero temperature, the uniform magnetization is activated as $m(h, T) \propto 1-\exp (-\beta h)$. In both cases, at sufficiently large temperatures, $m(h, T)$ exhibit Curie-like decay which is common to all Ising systems. At low temperatures, its behaviour depends on the field, beginning at 1 for $h>h_{z c}$ and terminating at 0 for $h<h_{z c}$. This behaviour at finite temperatures is illustrated in Figs. 5 and 6. Observe in Fig. 5 the gradual transition of the system into the ferromagnetic ground state as $h_{z}$ is increased. This shows that $h_{z}$ favours the ferromagnetic alignment of spins. On the contrary, as observed in Fig.6, an increase in temperature favours antiferromagnetic alignment of spins due to thermal agitations caused by temperature increase.

Table 1. Variation of the energies of the two-spin system with longitudinal field. At critical field of $h_{z c}=0.5$, the ground

\begin{tabular}{ccccc}
\multicolumn{5}{c}{ state shifts from $S_{\text {tot }}^{z}=1$ to $S_{\text {tot }}^{z}=0$} \\
\hline $\mathrm{h}_{\mathrm{Z}} / \mathrm{J}$ & $\mathrm{E}_{1}$ & $\mathrm{E}_{2}$ & $\mathrm{E}_{3}$ & $\mathrm{E}_{4}$ \\
\hline 0.00 & -0.25 & -0.25 & 0.25 & 0.25 \\
0.10 & -0.25 & -0.25 & 0.15 & 0.35 \\
0.20 & -0.25 & -0.25 & 0.05 & 0.45 \\
0.30 & -0.25 & -0.25 & -0.05 & 0.55 \\
0.40 & -0.25 & -0.25 & -0.15 & 0.65 \\
0.50 & -0.25 & -0.25 & -0.25 & 0.75 \\
0.60 & -0.25 & -0.25 & -0.35 & 0.85 \\
0.70 & -0.25 & -0.25 & -0.45 & 0.95 \\
0.80 & -0.25 & -0.25 & -0.55 & 1.05 \\
0.90 & -0.25 & -0.25 & -0.65 & 1.15 \\
1.00 & -0.25 & -0.25 & -0.75 & 1.25 \\
\hline
\end{tabular}

Table 2. Variation of the energies of the two-spin system with transverse field. The energy levels are nondegenerate.

\begin{tabular}{lcccc}
\hline$h_{x} / J$ & $\mathrm{E}_{1}$ & $\mathrm{E}_{2}$ & $\mathrm{E}_{3}$ & $\mathrm{E}_{4}$ \\
\hline 0.00 & -0.25 & 0.25 & -0.2500 & 0.2500 \\
0.10 & -0.25 & 0.25 & -0.2693 & 0.2693 \\
0.20 & -0.25 & 0.25 & -0.3202 & 0.3202 \\
0.30 & -0.25 & 0.25 & -0.3905 & 0.3905 \\
0.40 & -0.25 & 0.25 & -0.4717 & 0.4717 \\
0.50 & -0.25 & 0.25 & -0.5590 & 0.5590 \\
0.60 & -0.25 & 0.25 & -0.6500 & 0.6500 \\
0.70 & -0.25 & 0.25 & -0.7433 & 0.7433 \\
0.80 & -0.25 & 0.25 & -0.8382 & 0.8382 \\
0.90 & -0.25 & 0.25 & -0.9341 & 0.9341 \\
1.00 & -0.25 & 0.25 & -1.0308 & 1.0308 \\
\hline
\end{tabular}


Table 3. Variation of the energies of the two-spin system with mixed field.The antiferromagnetic alignment of spin is easily disrupted by mixed field.

\begin{tabular}{|c|c|c|c|c|}
\hline$h_{z}=h_{x}$ & $\mathrm{E}_{1}$ & $E_{2}$ & $E_{3}$ & $\mathrm{E}_{4}$ \\
\hline 0.1 & -0.26997 & -0.25 & 0.16142 & 0.35855 \\
\hline 0.2 & -0.32853 & -0.25 & 0.09923 & 0.47930 \\
\hline 0.3 & -0.41856 & -0.25 & 0.06149 & 0.60701 \\
\hline 0.4 & -0.52896 & -0.25 & 0.03997 & 0.73899 \\
\hline 0.5 & -0.65097 & -0.25 & 0.02748 & 0.87349 \\
\hline 0.6 & -0.77953 & -0.25 & 0.01985 & 1.00967 \\
\hline 0.7 & -0.91195 & -0.25 & 0.01494 & 1.14701 \\
\hline 0.8 & -1.04677 & -0.25 & 0.01161 & 1.28516 \\
\hline 0.9 & -1.18318 & -0.25 & 0.00927 & 1.42390 \\
\hline 1.0 & -1.32067 & -0.25 & 0.00757 & 1.56310 \\
\hline
\end{tabular}

Table 4. Varying $h_{x}$ at the critical longitudinal field ( $h_{z}=0.5$ ). The removal of degeneracy by transverse field is manifested in the shift of ground state from $E_{3}$ to $E_{1}$.

\begin{tabular}{ccccc}
\hline$h_{x}$ & $\mathrm{E}_{1}$ & $\mathrm{E}_{2}$ & $\mathrm{E}_{3}$ & $\mathrm{E}_{4}$ \\
\hline 0.0000001 & -0.250000 & -0.25 & -0.250000 & 0.75000 \\
0.0000010 & -0.250001 & -0.25 & -0.249999 & 0.75000 \\
0.1000000 & -0.323079 & -0.25 & -0.181921 & 0.75500 \\
0.2000000 & -0.400381 & -0.25 & -0.119611 & 0.76999 \\
0.3000000 & -0.481193 & -0.25 & -0.063724 & 0.79492 \\
0.4000000 & -0.564895 & -0.25 & -0.014671 & 0.82957 \\
0.5000000 & -0.650969 & -0.25 & 0.027479 & 0.87349 \\
0.6000000 & -0.738992 & -0.25 & 0.063022 & 0.92597 \\
0.7000000 & -0.828620 & -0.25 & 0.092556 & 0.98606 \\
0.8000000 & -0.919978 & -0.25 & 0.116858 & 1.05272 \\
\hline
\end{tabular}

Table 5. Quantum Spin flunctuations at the G.S. This is investigated when either $h_{z}$ or $h_{x}$ is fixed and the other varied.

\begin{tabular}{|c|c|c|c|c|c|}
\hline $\mathrm{h}_{\mathrm{z}}$ & $\mathrm{h}_{\mathrm{x}}$ & $\begin{array}{c}\mathrm{G} . \mathrm{S} \\
\left(\text { fixed } \mathrm{h}_{\mathrm{z}}\right)\end{array}$ & $\mathrm{h}_{\mathrm{x}}$ & $\mathrm{h}_{\mathrm{z}}$ & $\begin{array}{c}\mathrm{G} . \mathrm{S} \\
\left(\text { fixed } \mathrm{h}_{\mathrm{x}}\right)\end{array}$ \\
\hline 0.1 & 0.1 & -0.26997 & 0.1 & 0.1 & -0.26997 \\
0.1 & 0.2 & -0.32212 & 0.1 & 0.2 & -0.27243 \\
0.1 & 0.3 & -0.39336 & 0.1 & 0.3 & -0.27797 \\
0.1 & 0.4 & -0.47498 & 0.1 & 0.4 & -0.29082 \\
0.1 & 0.5 & -0.56245 & 0.1 & 0.5 & -0.32308 \\
& & & & & \\
\hline
\end{tabular}


Table 6. Variations of $\mathrm{m}(\mathrm{h}, \mathrm{T})$ with $\mathrm{h}$ for fixed values of $\mathrm{T}$. This table shows a gradual transition from antiferromagnetism to ferromagnetion when the longitudinal field is increased.

\begin{tabular}{ccccc}
\hline $\mathrm{h} / \mathrm{J}$ & $\mathrm{m}(\mathrm{h}, \mathrm{T})$ & $\mathrm{m}(\mathrm{h}, \mathrm{T})$ & $\mathrm{m}(\mathrm{h}, \mathrm{T})$ & $\mathrm{m}(\mathrm{h}, \mathrm{T})$ \\
$\mathrm{T}=0.04 \mathrm{~J}$ & $\mathrm{~T}=0.08 \mathrm{~J}$ & $\mathrm{~T}=0.1 \mathrm{~J}$ & $\mathrm{~T}=0.4 \mathrm{~J}$ \\
\hline 0.00 & 0.00000 & 0.00000 & 0.00000 & 0.00000 \\
0.50 & 0.99615 & 0.33333 & 0.03915 & 0.29782 \\
1.00 & 0.99999 & 0.99615 & 0.33331 & 0.62875 \\
1.50 & 0.99999 & 0.99999 & 0.85898 & 0.85810 \\
2.00 & 1.00000 & 0.99999 & 0.98670 & 0.95499 \\
2.50 & 1.00000 & 1.00000 & 0.99890 & 0.98670 \\
\hline
\end{tabular}

Table

7.Variations of $\mathrm{m}(\mathrm{h}, \mathrm{T})$ with $\mathrm{T}$ for fixed values of $\mathrm{h}$. This table shows a gradual transition from ferromagnetism to antiferromagnetion when the temperature is increased.

\begin{tabular}{lllll}
\hline $\mathrm{J} / \mathrm{T}$ & $\mathrm{m}(\mathrm{T}, \mathrm{h})$ & $\mathrm{m}(\mathrm{T}, \mathrm{h})$ & $\mathrm{m}(\mathrm{T}, \mathrm{h})$ & $\mathrm{m}(\mathrm{T}, \mathrm{h})$ \\
$\mathrm{h}=0.8 \mathrm{~J}$ & $\mathrm{~h}=1.6 \mathrm{~J}$ & 0.00000 & 0.00000 \\
& 0.00000 & 0.00000 & 0.00000 & 0.50495 \\
\hline 0.0 & 0.17367 & 0.33878 & 0.41569 & 0.77756 \\
0.5 & 0.29741 & 0.56211 & 0.67029 & 0.90848 \\
1.0 & 0.38432 & 0.71230 & 0.82217 & 0.96458 \\
1.5 & 0.44858 & 0.81612 & 0.90886 & 0.98670 \\
2.0 & 0.50008 & 0.88608 & 0.95499 & 0.99507 \\
2.5 & 0.54452 & 0.93118 & 0.97825 & 0.99933 \\
\hline .0 & 0.62239 & 0.97603 & 0.99507 & \\
\hline
\end{tabular}

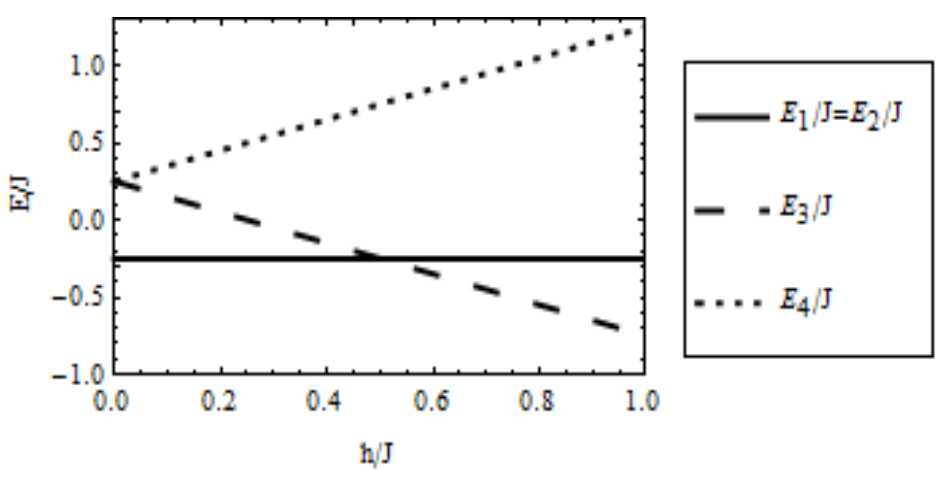

Fig. 2. Energy levels of two-spin Ising system as a function of the external longitudinal field. Level crossing occurs at $h_{z}$ $/ \mathrm{J}=0.5$. 


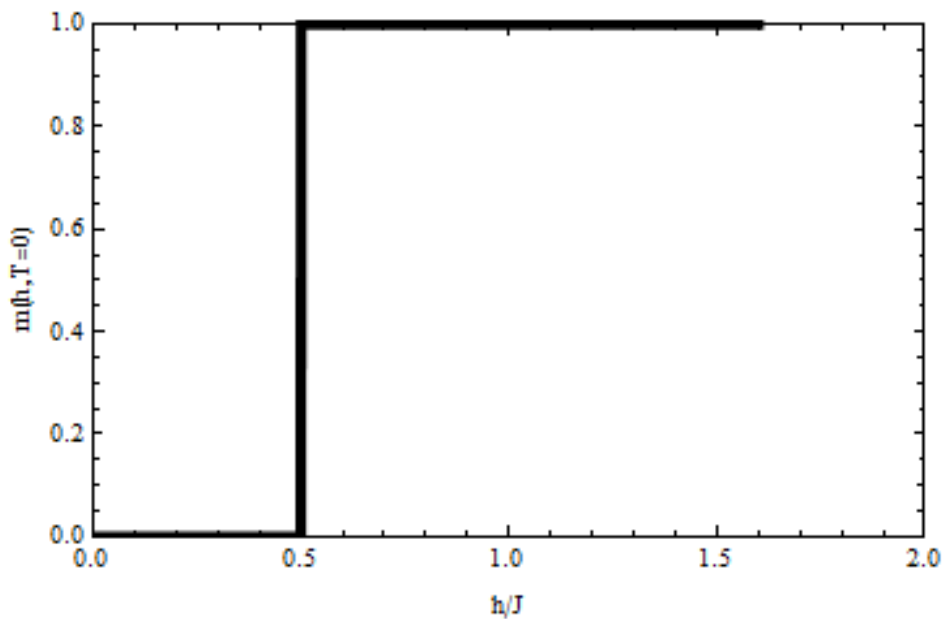

Fig. 3. Quantum of magnetization at zero temperature $(\mathrm{m}(\mathrm{h}, \mathrm{T}=0))$ of two-spin Ising system. The spins will remain antialigned until a critical field of $h_{z}=J / 2$ is reached.

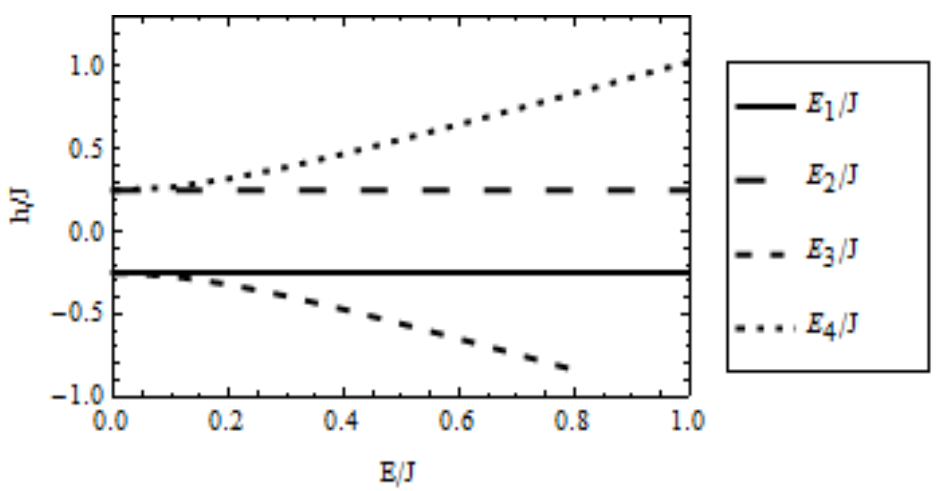

Fig. 4. Energy levels of two-spin Ising system as a function of external transverse field. All degeneracies are completely removed and level crossing occurs at an infinitesimal field.

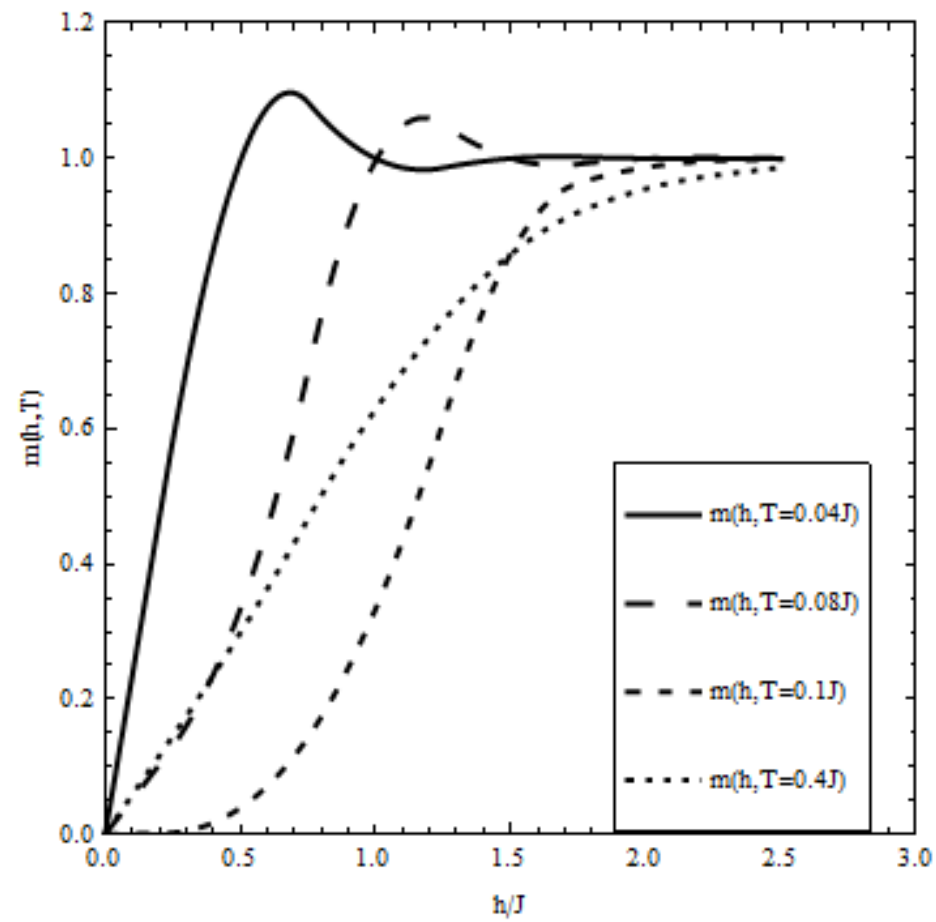

Fig .5. Magnetic field dependence of the uniform magnetization of a 2-site Ising system. This dependence is investigated for given values of $\mathrm{T}$. Thermal fluctuations smooth out the step function seen in $\mathrm{m}(\mathrm{h}, \mathrm{T}=0)$. 


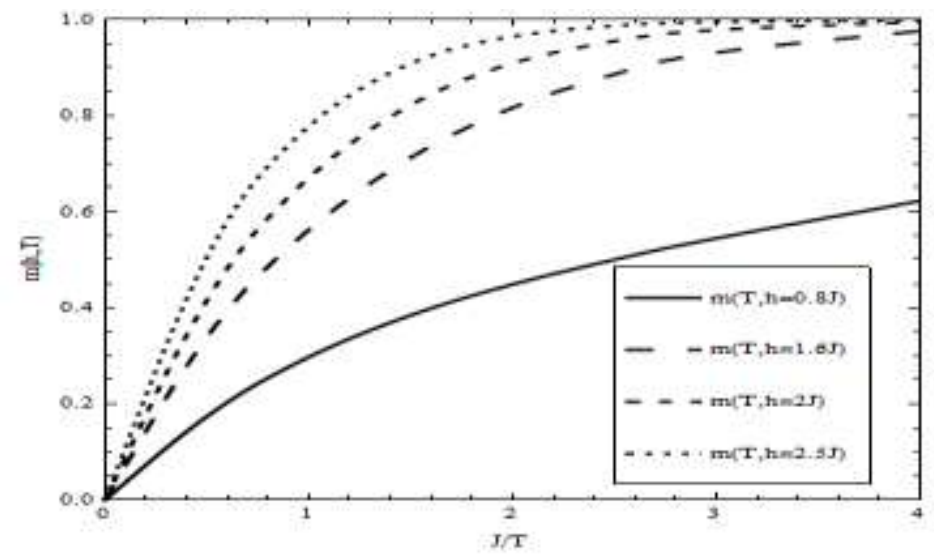

Fig 6. Temperature dependence of the uniform magnetization of a 2-site Ising system. This dependence is investigated for given values of $h$. Thermal fluctuations smooth out the step function seen in $m(h, T=0)$.

\section{Conclusion}

The magnetic properties of two-spin system are studied within the Ising quantum antiferromagnetic model. At the critical longitudinal magnetic fields of $\mathrm{h}_{\mathrm{zc}}=\mathrm{J} / 2$, the system is found to undergo a quantum phase transition (QPT) to the ferromagnetic state. The effect of transverse field on the spin system is so strong that an infinitesimal departure from zero transverse field causes the system to undergo a QPT to ferromagnetic state. This shows that quantum spin fluctuations are more pronounced in the transverse field than the longitudinal field. It is also observed that QPT for the special case of mixed fields occurs at a faster rate than the separate effect of longitudinal and transverse fields.

The quantum magnetization observed at zero temperature for the Ising spin-two systems is found to be completely wiped out at finite temperatures due to thermal fluctuations. A temperature increase favours antiferromagnetic alignment of spins, while a decrease in temperature favours ferromagnetic alignment. On a macroscopic view, this temperature increase accounts for the disappearance of magnetic field when a magnet is subjected to thermal heating in accordance with curie's law. The experimental evidence of this QPT has been observed at optimum doping in the cuprate $\mathrm{Bi}_{2} \mathrm{Sr}_{2-x} \mathrm{La}_{x} \mathrm{CuO}_{6+\delta}$ and in quasi-one dimensionally Ising ferromagnet [10,30]. Ultracold atoms in optical lattices has also provided a versatile tool with which to investigate fundamental properties of quantum many-body systems such as quantum phase transition and quantum spin dynamics [39].

\section{References}

[1]. Elliott R. J., Pfeuty P., and Wood C. (1970). Ising Model with a Transverse Field. Phys. Rev. Lett. 25, $443-446$

[2]. Sachdev S. and Ye J. (1992). Universal quantum-critical dynamics of two-dimensional antiferromagnets. Phys. Rev. Lett. 69, 24112414.

[3]. Sachdev S and Young A. P. (1997). Low Temperature Relaxational Dynamics of the Ising Chain in a Transverse Field, Phys. Rev. Lett. 78, 2220-2223

[4]. Sachdev S. (1999). Quantum Phase Transitions, Cambridge University Press, Cambridge U.K.

[5]. Sachdev S. (2000). Quantum Criticality: Competing Ground States in Low Dimensions. Science 288, 475-480

[6]. Ovchinnikov A.A., Dmitriev D.V., and Krivnov V.Y. (2003). The antiferromagnetic Ising chain in a mixed transverse and longitudinal magnetic field. Phys. Rev. B 68, $214406-1-10$

[7]. Vojta M.,. (2003). Quantum phase transitions. Rep. Prog. Phys. 66, 2069-2110.

[8]. Coleman P. and Schofield A. J. (2005). Quantum criticality. Nature vol 433, 226-229.

[9]. Zhang J., Cucchietti F. M., Chandrashekar C. M., Laforest M., Ryan C. A., Ditty M., Hubbard A., Gamble J.K., and Laflamme R. Direct observation of quantum criticality in Ising spin chains. (2009). Phys. Rev. A 79, 012305 -1-12

[10]. Coldea R.,Tennant D.A., Wheeler E.M., Wawrzynska E., Prabhakaran D., Telling M., Habicht K., Smeibidl P. and Kiefer K.(2010). Quantum criticality in an Ising chain: Experimental evidence for the emergent E8 symmetry. Science, 327, 177-180.

[11]. Sachdev S. (2011). Quantum criticality . Physics Today 64, 29-35.

[12]. Haldane F. D .M. 1981). Luttinger liquid theory' of one-dimensional quantum fluids. I. Properties of the Luttinger model and their extension to the general 1D interacting spinless Fermi gas. J. Phys. C: Solid State Phys. 14, 2585-2609.

[13]. Adilet I. and Leonid I. G. (2009). Universal Theory of Nonlinear Luttinger Liquids. Science 9, Vol. 323 no. 5911 pp. $228-231$.

[14]. Laroche D., Gervais G., Lilly M. P. and Reno J. L. (2014). 1D-1D Coulomb Drag Signature of a Luttinger Liquid. Science 7, Vol. 343 no. $6171,631-634$

[15]. Mott. N.F. (1949). The Basis of electron theory of metals, with special reference to the transition metals. Proc. Phys.Soc.62, 7-A; 416-422.

[16]. Anderson P. W. (1961). Localized Magnetic States in Metals, Phys. Rev. 124, 41-53.

[17]. Byczuk K., Hofstetter W., and Vollhardt D. (2010). Anderson localization vs. Mott-Hubbard metal-insulator transition in disordered, interacting lattice fermion systems. Int. J. Mod. Phys. B 24, 1727.

[18]. Chowdhury D., Swingle B., Berg E., and Sachdev S. (2013). Singularity of the London penetration depth at quantum critical points in superconductors. Phys. Rev. Lett. 111, 157004-1-15

[19]. Punk M. and Sachdev S. (2013). Mobile impurity near the superfluid-Mott-insulator quantum critical point in two dimensions. Phys. Rev. A 87, 033618-1-13.

[20]. Sachdev S., Bernhard K. (2011). Quantum Criticality Physics Today vol 64, no.2, pp29.

[21]. Ehika S. and Idiodi J.O.A. (2012). Quantum phase transition in the Heisenberg model: A case study of a two-spin system. Journal of NAMP Vol. 21, pp. 11-20. 
[22]. Pasquale C., Fabian H. L. E, and Maurizio F. (2011). Quantum Quench in the Transverse-Field Ising Chain. Phys. Rev. Lett. 106, 227203-1-4.

[23]. de Oliveira T. R., Rigolin G., de Oliveira M. C., and Miranda E. (2006). Multipartite Entanglement Signature of Quantum Phase Transitions . Phys. Rev. Lett. 97, 170401-1-4.

[24]. Osterloh A., Amico L., Falci G., and Fazio R., (2002). Scaling of Entanglement close to a Quantum Phase Transition. Nature 416, pp608-610.

[25]. Osborne T. J. and Nielsen M. A. (2002). Entanglement in a simple quantum phase transition. Phys. Rev. A 66, 032110-1-14 .

[26]. Schützhold R. and Schaller G. (2006). Adiabatic quantum algorithms as quantum phase transitions: First versus second order . Phys. Rev. A 74, 060304(R) -1-4.

[27]. Latorre J. I. and Orús R. (2004). Adiabatic quantum computation and quantum phase transitions Phys. Rev. A 69, 062302-1-5.

[28]. Zanardi P., Paris M. G. A. and Venuti L. C. (2008). Quantum criticality as a resource for quantum estimation. Phys. Rev. A 78, 042105-1-7

[29]. Invernizzi C., Korbman M., Venuti L.C. and Paris M. G. A. (2008). Optimal quantum estimation in spin systems at criticality. Phys. Rev. A 78, 042106-1-7

[30]. Balakirev F. F., Betts J.B., Migliori A., Ono S., Ando Y. and Boebinger G.S. (2003). Signature of optimal doping in Hall-effect measurements on a high-temperature superconductor. Nature, 424, 912-915.

[31]. Ando Y., Ono S., Sun X. F., Takeya J., Balakirev F. F., Betts J. B. and Boebinger G.S. (2004). Quantum Phase Transitions in the Cuprate Superconductor $\mathrm{Bi}_{2} \mathrm{Sr}_{2-x} \mathrm{La}_{x} \mathrm{CuO}_{6+\delta}$ Phys. Rev. Lett. 92, pp247004-4

[32]. Bookjans E. M., Vinit A. and Raman C. (2011). Quantum Phase Transition in an Antiferromagnetic Spinor Bose-Einstein Condensate. Phys. Rev. Lett.107, 195306-1-5.

[33]. Sachdev S. (2008). Quantum magnetism and criticality. Nat. Phys. 4, 173-185.

[34]. Greiner M., Mandel O., Esslinger T., Hänsch T. W. and Bloch I. (2002).Quantum phase transition from a superfluid to a Mott insulator in a gas of ultracold atoms. Nat 415, 39-44.

[35]. Wölfle P. and Schmitteckert P.( 2015). Quantum phase transitions in frustrated magnetic systems, Eur. Phys. J. Special Topics 224, 1087-1103.

[36]. Tsvelik A. M. and Yevtushenko O. M. (2015). Quantum Phase Transition and Protected Ideal Transport in a Kondo Chain, Phys Rev. Lett. 115, 216402-1-216402-5.

[37]. Gómez-Ruiz F. J., Mendoza-Arenas J. J., Rodr'1guez F. J., Tejedor C., and Quiroga L. (2016). Quantum phase transitions detected by a local probe using time correlations and violations of Leggett-Garg inequalities, Phys. Rev B 93, 035441-1—035441-10.

[38]. Toyoda K., Matsuno Y., Noguchi A., Haze and Urabe S. (2013).Experimental Realization of a Quantum Phase Transition of Polaritonic Excitations. Phys. Rev. Lett 111, 160501-1-5.

[39]. Weitenberg C., Endres M., Sherson J. F, Cheneau M., Schauß P., Fukuhara T., Bloch I., and Kuhr S. (2011). Single-spin addressing in an atomic Mott insulator. Nature 471, pp319-324. 